\title{
If we are what we Eat, How have Decreases in Nutritional Densities of Food Affected Health?
}

\author{
Leonard Sonnenschein* \\ Author of understanding Cellular Metabolism: Nutrition, Health and Beauty, President of World Aquarium and Conservation for the Oceans Foundation, USA
}

Received: July 03, 2017; Published: July 10, 2017

*Corresponding author: Leonard Sonnenschein, President of World Aquarium and Conservation for the Oceans Foundation, Author of Understanding Cellular Metabolism: Nutrition, Health and Beauty, 810 Lumiere Place Blvd., St. Louis, Missouri 63102 USA, Tel: 314-609-2798; Email: LSAQUAMAN@AOL.COM, Website: www.childrensaquarium.org

\section{Introduction}

Since 1950, the overall nutritional density of crops has significantly decreased. Variations in minerals and vitamins can be directly attributed to: increased productivity = decreased nutritional values, increased soil use without fertilizers = less fertile soils, and choice of cultivars. This change in nutritional density means that in order to get more nutrients from what we eat means we need to eat more, make changes in agricultural practices, and/or adapt a supplement program that insures delivery of active ingredients.

\section{History}

In 1992, we began looking at the nutritional requirements of coral and the importance of the presence of nutrients. From coral nutritional data collected over twenty years it was found that these findings applied also to plants, aquatic and land animals and can determine transfer mechanisms and interactions of $\mathrm{pH}$ and thermal thresholds. These laboratory studies led to conclusive findings that principles of cellular absorption and metabolism of nutrients with merit more broadly to plant, animal, coral and fish applications. As it turns out the availability of nutrients within a solution is directly related to the health of coral species. Coral growth can be increased by changing nutrients that are essential for promoting growth. The same can be said for human nutrition and human metabolism being related to the presence of nutrients that are easily absorbed as in the case of the aqueous osmotic absorption for corals these nutrients that can be more cell available. For the human cells, increases in nutritional solution density allows for improved adsorption, better growth, better metabolism and overall better health for the cell.

\section{Improved Active Ingredient Delivery}

Recent independent mouse studies conducted at GVK Laboratories in Hyderabad India showed that adding an organic natural adjuvant to the food can allow for up to 10 fold increased absorption of Vitamin D3 as an active ingredient. By increasing the availability of important nutrients both wastes of non-metabolized active ingredients and improving the important active ingredient delivery can help for maintenance of metabolism.

As our average age increases the dependency upon nutritional supplements increases. It is important to ensure appropriate vitamin and mineral formulations address active ingredients uptake and that public awareness needs to be forthright in this process.

\section{Foods and Nutrition}

The nutrient density of the foods that we eat makes a big difference in terms of the actual adsorption of nutrients. Nutrients in the food provide the highest degree of absorption of those active ingredients because of the natural state of the nutrients in the food compared to nutrients within a formulary compound as how much nutrients are actually adsorbed. Malnutrition can occur because of not enough food to eat, but also is a consequence of not getting the nutrients to the body because the nutritional quality of what we are eating is not sufficient to supply the metabolic needs. In some African countries the nutritional value of some crops as food is not much different than eating cardboard.

\section{Conclusion}

It is clear that we are what we eat and that our health is directly related to our nutrition. Minerals that are most important for our immune system such as Iodine, Iron, Calcium and Zinc have been significantly depleted from crops.

Vitamins such as A, B and C have been depleted as much as $80 \%$ from foods such as oranges when compared to prior to 1960 . It is likely that these deficiencies have lead to poorer health as is especially concomitant in areas where agriculture is most perilous such as in African countries where soil health and crop yields are marginal. Nutritional density of crops drops as soil is used and not replenished. There is great need to provide new solutions to improve nutritional density of crops, increase public awareness of best nutritional supplements, and show better nutrition overall can lead to improved health. 


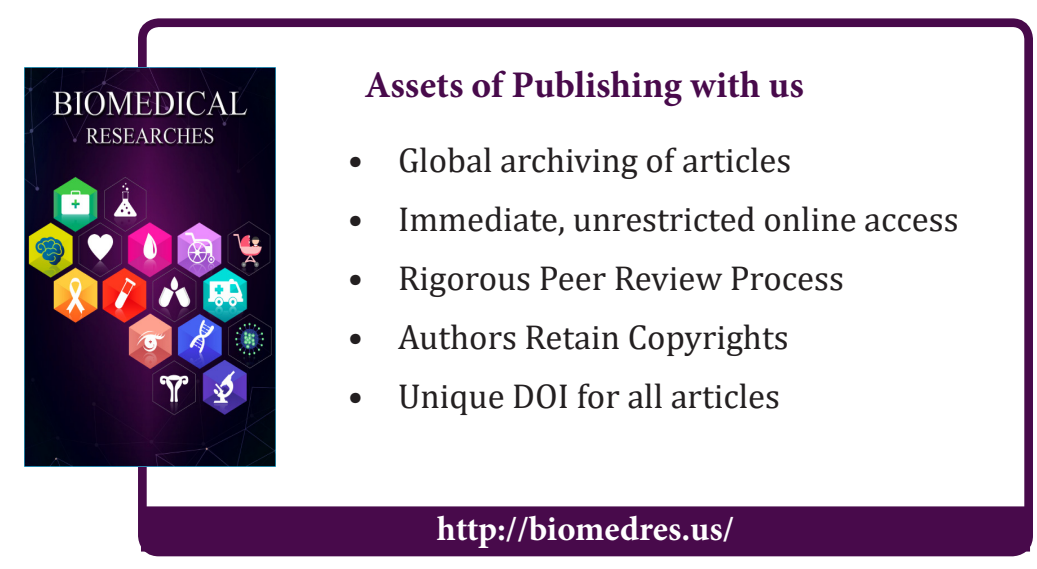

